\title{
Engineering the Evaluation Approach to Fit Different Web Project and Organization Needs
}

\author{
Luis Olsina \\ GIDIS_Web, Engineering School at Universidad Nacional de La Pampa, Argentina \\ olsinal@ing.unlpam.edu.ar
}

\begin{abstract}
Web applications, among other software entities, and their quality evaluation has been the subject of abundant research; however, open issues still remain. This tutorial discusses a general measurement and evaluation approach which is based on both a quality modeling framework and strategies. An evaluation strategy is in turn grounded on three principles namely: a conceptual framework, a well-established process, and methods/tools. The illustrated evaluation approach allows instantiating evaluation strategies -reusing these three principles- to engineering different organization-level information needs.
\end{abstract}

\section{$1 \quad$ Introduction}

Measurement, evaluation, analysis and recommendation are support processes to primary web engineering processes, i.e. by giving support to deal with information needs at different project or organizational levels. In addition, quality is one out of four main dependent variables for managing web projects. For each engineered project, and independently of the development/maintenance lifecycle adopted, levels of quality for its entities and attributes should be agreed, specified, measured and evaluated for analyzing and improving them. To assure repeatability and consistency of results for better analysis and decision making, it is necessary to have a welldefined yet customizable evaluation approach.

This tutorial discusses a general measurement and evaluation $(M \& E)$ approach which is based on two main pillars, namely: i) a quality modeling framework; and ii) $M \& E$ strategies, which in turn are grounded on three principles viz. a $M \& E$ conceptual framework, a well-established $M \& E$ process, and evaluation methods and tools. The $M \& E$ conceptual framework capability should be built on a robust terminological base, e.g. an ontology, which explicitly and formally specifies the main concepts, properties, relationships, and constraints for the M\&E domain, as well as their grouping into components. This principle ensures terminological uniformity among the other capabilities and thus the consistency of results. The second principle is the $M \& E$ process [1], which describes what to do, by specifying the activities to be planned and executed, their inputs and outputs, roles, interdependencies, among other aspects. A well-established M\&E process not only facilitates the understanding and communication among stakeholders but also ensures repeatability and reproducibility in the implementation of the activities. Lastly, methods and tools, which enable to 
perform and automate the activities' descriptions. Methods are allocated in a flexible way to perform the specified activities and usually automated by tools.

This general M\&E approach can be adapted to fit different organizational levels and information needs for different quality focuses regarding entities categories such as resource, product, system, system in use, etc. in a flexible yet structured manner.

The development of this tutorial makes use of both theoretical and practical background. From the practical point of view, so far, we have developed two M\&E strategies, namely: GOCAME (Goal-Oriented Context-Aware Measurement and Evaluation) [3], and SIQinU (Strategy for understanding and Improving Quality in Use) [2], which the latter was used in a testing industry case. These strategies can be instantiated regarding the quality modeling framework and specific information needs.

\section{$2 \quad$ Learning Objectives}

To summarize, the main tutorial learning objectives are: 1) Review background concepts such as information need, quality and entity category; quality models, and strategies regarding the M\&E process, conceptual framework, method/tool; 2) Get insight on how the quality modeling framework can be instantiated in a purposeful way not only for understanding but also for improvement, using for this end the customized strategy. The learning aim is to see that many different strategies can be instantiated from the same quality modeling framework, regarding different information needs and organizational levels; and 3) Understand how a concrete strategy for improving a web application (e.g. its external quality and quality in use) can be used, while excerpts of a real case study are illustrated.

\section{References}

1. Becker, P., Lew, P., Olsina, L.: Specifying Process Views for a Measurement, Evaluation and Improvement Strategy. Advances in Software Engineering Journal 2012, 28 (2012), http: / / www.hindawi.com/journals/ase/contents /

2. Lew, P., Olsina, L., Becker, P., Zhang, L.: An Integrated Strategy to Understand and Manage Quality in Use for Web Applications. Requirements Engineering Journal 16(3), 1-32 (2011)

3. Olsina, L., Lew, P., Dieser, A., Rivera, B.: Using Web Quality Models and a Strategy for Purpose-Oriented Evaluations. Journal of Web Engineering 10(4), 316-352 (2011) 